\title{
The evolution of concerted evolution
}

\author{
Laurence D. Hurst ${ }^{*}$ and Nicholas G. G. Smith
}

Department of Biology and Biochemistry, Centre for Mathematical Biology, University of Bath, Claverton Down, Bath BA2 7AY, UK

\begin{abstract}
Concerted evolution is a consequence of processes that convert copies of a gene in a multigene family into the same copy. Here we ask whether this homogenization may be adaptive. Analysis of a modifier of homogenization reveals (1) that the trait is most likely to spread if interactions between deleterious mutations are not strongly synergistic; (2) that selection on the modifier is of the order of the mutation rate, hence the modifier is most likely to be favoured by selection when the species has a large effective population size and/or if the modifier affects many genes simultaneously; and (3) that linkage between the genes in the family, and between these genes and the modifier, makes invasion of the modifier easier, suggesting that selection may favour multigene families being in clustered arrays. It follows from the first conclusion that genes for which mutations may often be dominant or semi-dominant should undergo concerted evolution more commonly than others. By analysis of the mouse knockout database, we show that mutations affecting growth-related genes are more commonly associated with dominant lethality than expected by chance. We predict then that selection will favour homogenization of such genes, and possibly others that are significantly dosage dependent, more often than it favours homogenization in other genes. The first condition is almost the opposite of that required for the maintenance of sexual reproduction according to the mutationdeterministic theory. The analysis here therefore suggests that sexual organisms can simultaneously minimize both the effects of deleterious, strongly synergistically, interacting mutations and those that interact either weakly synergistically, multiplicatively, or antagonistically, assuming the latter class belong to a multicopy gene family. Recombination and an absence of homogenization are efficient in purging deleterious mutations in the former class, homogenization and an absence of recombination are efficient at minimizing the costs imposed by the latter classes.
\end{abstract}

Keywords: evolution of sex, evolution of ploidy, gene conversion, epistatic interactions, multigene arrays

\section{INTRODUCTION}

Natural selection has been evoked to explain many features of the structure of genes and genomes; what, for want of a term, might be called 'evolutionary genomics'. For example, selectionist explanations have been provided to account for why some genes might be (i) in multiple copies and others in just a single copy (e.g. Haig 1993; Hurst 1992, 1996), (ii) on one chromosome rather than another (e.g. Hurst 1994; Rice 1992), (iii) on particular parts of a chromosome (e.g. Charlesworth \& Hartl 1978), and (iv) why they should be linked to certain other genes (e.g. Bodmer \& Parsons 1962; Hutson \& Law 1993; Korol et al. 1994; Lyttle 1991). Here we ask whether, and under what conditions, the ability to undergo concerted evolution might be an adaptive feature of genomes.

Many multigene families undergo various processes, such as gene conversion and unequal crossing over, that convert copies of the gene into identical copies. Such homogenized families can go to fixation within a species and cause divergence between species. As a consequence, all the copies within the species appear to be 'coordinated' in their evolution and hence the families are said to undergo concerted evolution (reviewed in Elder \& Turner 1995). Why should this happen?

*Author for correspondence (1.d.hurst@bath.ac.uk).
The population genetics of homogenization has attracted considerable attention (reviewed in Weir et al. 1985). For the most part these analyses examine the population genetic consequences of homogenization. For example, Ohta (1989) considered the changes to the populational load that occur as a consequence of homogenization. She concludes that homogenization always reduces the load. This suggests that competition between reproductively isolated populations, some with homogenization and some without, might typically be won by the populations with concerted evolution. Here we ask a different question, namely whether homogenization might be adaptive at the individual level. More precisely, we ask whether, when a modifier allowing members of a gene family to be homogenized appears in a population, it will spread because of its effect on the gene family.

\section{WHY HOMOGENIZATION MAY BE ADAPTIVE}

Imagine, for simplicity, a haploid organism with a gene family comprising two genes. Deleterious mutations occur in the genes at some very low rate. Imagine then that one copy has mutated but the other has not. We can now ask what the organism should do. We shall assume that the organism cannot tell which is the mutated copy and which is the wild-type copy. If it could tell, then replacing the mutated with the unmutated copy will always be 
advantageous, assuming this to be an error-free process (cf. Bengtsson 1990).

The organism has a choice: either to do nothing, or to randomly pick one of the two copies and replace the other copy with the chosen copy. At an extreme, if two copies of the mutated copy do you no more harm than one mutated copy, then one can only ever gain (in terms of immediate fitness effects) by randomly selecting one of the two copies and replacing the other with this copy. Half the time fitness goes up, half the time fitness stays the same. At the other extreme, if one copy of the mutant does you no harm, but two copies are very deleterious, then it is better, in the short-term, not to risk the production of an array with two mutant copies. Whether homogenization has the effect of immediately increasing or decreasing fitness is therefore dependent on the nature of the fitness interaction between the two copies of the gene.

More generally, we may consider that the fitness of a haploid individual with two mutant versions of the gene is $(1-s)^{2}+\epsilon$, when $1-s$ is the fitness of the individual with one mutated copy and one wild-type copy (cf. Otto \& Feldman 1997). When $\epsilon=0$, interactions are independent, i.e. fitness is multiplicative. For $\epsilon>0$, there is positive epistasis (alias antagonistic epistasis). If $\epsilon<0$, there is negative or synergistic epistasis. If $\epsilon>-s^{2}$, then we may say that there is weak synergistic epistasis. For the simple twolocus haploid case, it is trivial to show that conversion is immediately advantageous to the individual so long as $\epsilon>-s^{2}$. Random homogenization of the $A a$ gene pair will result in an individual with a fitness of 1 , half the time, and a fitness of $(1-s)^{2}+\epsilon$, half the time. The average fitness then will be $1-s+\left(s^{2}+\epsilon\right) / 2$.

This fitness is higher than that associated with not doing anything (fitness $1-s$ ) if $\epsilon>-s^{2}$, i.e. interactions must be weakly synergistic, multiplicative or antagonistic.

A fuller consideration requires analysis of a modifier locus controlling the tendency to convert one gene into another. It is this analysis that we provide below. The modifier could in principle be almost anything: a gene necessary for gene conversion, one affecting recombination rates, if recombination is necessary for homogenization, or simply a structural feature of the DNA of the genes concerned.

\section{THE MODEL}

Consider a sexual haploid organism (perhaps like Chlamydomonas). At an array with two copies of a gene in it, mutation and conversion can take place. We shall start by assuming that recombination does not occur between the two genes in the array. Three states exist for the array: wild-type $(A A)$, one copy is mutant $(A a)$, both copies are mutant $(a a)$. At a second locus, two alleles exist. $M$ is the modifier that allows the organism to select one of the two copies and replace the other copy with the selected copy. The wild-type allele $m$ is effectively a null allele. Note that the choice of which allele to select need not be random. So long as bias in selection of which copy to use does not covary with the probability of mutation, such a bias has no effect on the process.

\section{(a) Step 1. Sex and recombination}

Conversion we shall assume happens just prior to zygote formation. We assume for simplicity that in the presence of the modifier conversion always occurs. Whilst this is an unrealistic assumption, it should not greatly affect the qualitative conclusions. Gametes are then of five types: $M A A$ at frequency $x_{1}, M a a$ at frequency $x_{2}, m A A$ at frequency $x_{3}, m A a$ at frequency $x_{4}$ and maa at frequency $x_{5}$. Zygotes form by random mating. After zygote formation, meiosis occurs, and recombination can occur between the modifier and the array at a rate $r$. Recombination can generate a new form of haploid individual, i.e. one that is $M A a$. From the frequencies $x_{i}$, it is possible to calculate the frequencies of these haploid types. These we can label $x_{i a}$, where $x_{6 a}$ is the frequency of $M A a$. These frequencies are:

$$
\begin{aligned}
& x_{1 a}=x_{1}\left(x_{1}+x_{2}+x_{3}+(1-r)\left(x_{4}+x_{5}\right)\right)+r x_{2} x_{3} \\
& x_{2 a}=x_{2}\left(x_{1}+x_{2}+x_{5}+(1-r)\left(x_{3}+x_{4}\right)\right)+r x_{1} x_{5} \\
& x_{6 a}=r x_{4}\left(x_{1}+x_{2}\right) \\
& x_{3 a}=x_{3}\left(x_{1}+x_{3}+x_{4}+x_{5}+(1-r) x_{2}\right)+r x_{1}\left(x_{4}+x_{5}\right) \\
& x_{4 a}=x_{4}\left(x_{3}+x_{4}+x_{5}+(1-r)\left(x_{1}+x_{2}\right)\right) \\
& x_{5 a}=x_{5}\left(x_{2}+x_{3}+x_{4}+x_{5}+(1-r) x_{1}\right)+r x_{2}\left(x_{3}+x_{4}\right) .
\end{aligned}
$$

\section{(b) Step 2. Selection between haploid types}

Selection then affects the viability of the six haploid types. We assume that all mutations are deleterious. There are two ways to represent viabilities. Either, as above, to suppose that those that are $A A$ are of fitness 1, those that are $A a$ are of fitness $1-s$, and those that are $a a$ have fitness $(1-s)^{2}+\epsilon$. Alternatively, we could consider viabilities $1,1-H s$ and $1-s, H$ here being a parameter comparable to the penetrance, $h$, of a mutation at one locus in a diploid model. Given that we wish to compare the results from this analysis with data on dominance in real genes, the latter formulation is preferable. The modifier is associated with a cost, $\varphi$. After normalization, to take account of mean populational fitness, the following new frequencies, $x_{i b}$ can be found:

$$
\begin{aligned}
& x_{1 b}=\frac{x_{1 a}(1-\varphi)}{\bar{W}}, x_{2 b}=\frac{x_{2 a}(1-\varphi)(1-s)}{\bar{W}}, \\
& x_{6 b}=\frac{x_{6 a}(1-\varphi)(1-H s)}{\bar{W}}, x_{3 b}=\frac{x_{3 a}}{\bar{W}}, \\
& x_{4 b}=\frac{x_{4 a}(1-H s)}{\bar{W}}, x_{5 b}=\frac{x_{5 a}(1-s)}{\bar{W}},
\end{aligned}
$$

where $\bar{W}$, the mean fitness, is the sum of the numerators.

\section{(c) Step 3. Mutation}

Mutation can occur at a rate $\mu$. We shall assume that the mutation rate is so low that the probability that two copies of the gene can mutate can be ignored. This being so, the frequency of $A A$ gametes is reduced by a factor $(1-2 \mu)$, the frequency of $A a$ gametes is reduced by a factor $(1-\mu)$. These latter gametes also increase through mutation of $A A$ and $a a$ gametes increase in frequency due to mutation of $A a$. The new haploid frequencies $\left(x_{i c}\right)$ can be expressed as functions of $x_{i b}$ :

$$
\begin{aligned}
& x_{1 c}=x_{1 b}(1-2 \mu), \quad x_{2 c}=x_{2 b}+\mu x_{6 b}, \\
& x_{6 c}=x_{6 b}(1-\mu)+2 \mu x_{1 b}, \\
& x_{3 c}=x_{3 b}(1-2 \mu), \quad x_{4 c}=x_{4 b}(1-\mu)+2 \mu x_{3 b}, \\
& x_{5 c}=x_{5 b}+\mu x_{4 b} .
\end{aligned}
$$




\section{(d) Step 4. Conversion}

Finally, if the gametes bear the $M$ allele, conversion, by whatever mechanism, occurs. As noted above, we assume that if the modifier exists conversion will always occur. We can then provide an expression for the frequency of each gamete type in the next generation $\left(x_{i}^{\prime}\right)$ as a function of $x_{i c}$ :

$x_{1}^{\prime}=x_{1 c}+\frac{x_{6 c}}{2}, x_{2}^{\prime}=x_{2 c}+\frac{x_{6 c}}{2}$,

$x_{3}^{\prime}=x_{3 c}, x_{4}^{\prime}=x_{4 c}, \quad x_{5}^{\prime}=x_{5 c}$.

We therefore have an expression for the change in haploid type frequency as a function of its initial value, the mutation rate, the action of selection, and the presence or absence of conversion.

\section{MUTATION-SELECTION EQUILIBRIUM}

In the absence of the modifier $\left(x_{1}=x_{2}=0\right)$, the three array types will move to a mutation-selection equilibrium. Simultaneously, solving $x_{3}^{\prime}=x_{3}, x_{4}^{\prime}=x_{4}$ and $x_{5}^{\prime}=x_{5}$, under the condition that $\mathrm{x}_{3}+x_{4}+x_{5}=1$, we can obtain the equilibrium frequencies, these being:

$$
\begin{aligned}
& x_{3}^{*}=\frac{(s-2 \mu)(\mu-H s(1-\mu))}{s((H(2+s)-1)-H s)}, \\
& x_{4}^{*}=\frac{2 \mu(2 \mu-s)}{s(H((2+s)-1)-H s)}, \\
& x_{5}^{*}=\frac{2 \mu^{2}(H s-1)}{s((H(2+s)-1)-H s)} .
\end{aligned}
$$

A mutation-selection equilibrium exists so long as $\mu>0$ and $s>2 \mu$. The mean population fitness is $1-2 \mu$.

\section{THE BEHAVIOUR OF THE MODIFIER}

\section{(a) The cost-free modifier}

We shall start by considering the case of the neutral modifier $(\varphi=0)$. The conditions for the invasion of the modifier can be obtained from determining the solution of $\lambda>1$, where $\lambda$ is the leading eigenvalue of the linearized recursion equation for $x_{1}^{\prime}$ and $x_{2}^{\prime}$. For our case,

$\lambda=a+d-a d+b c$

where

$$
\begin{aligned}
& a=\frac{(1-\mu)\left(x_{3}^{*}+x_{4}^{*}\left(1-\frac{r(1+H s)}{2}\right)+x_{5}^{*}(1-r)\right)}{\bar{W}} \\
& b=\frac{r(1-\mu)\left(x_{3}^{*}+x_{4}^{*}\left(\frac{1-H s}{2}\right)\right)}{\bar{W}} \\
& c=\frac{\mu x_{3}^{*}+x_{4}^{*}\left(\mu+\frac{r(1-m-H s(1+\mu)}{2}\right)+x_{5}^{*}(\mu(1-r)}{+r(1-s))} \\
& \bar{W}
\end{aligned}
$$

$$
\begin{aligned}
& d= \\
& x_{3}^{*}(1-r(1-\mu)-s(1-r))+ \\
& \frac{x_{4}^{*}\left(1-s(1-r)-\frac{r(1-\mu+H s(1+\mu))}{2}\right)+x_{5}^{*}(1-s)}{\bar{W}}
\end{aligned}
$$

and where $\bar{W}$ is the mean population fitness at mutationselection equilibrium. The condition resolves to:

$r<\frac{\mu-H(2 \mu-s+\mu s)}{(1-\mu)(1-H(2-s))}$.

A graphical representation of this is given in figure 1. Two special cases are noteworthy. If the modifier is in perfect linkage with the array being modified $(r=0)$, then we obtain the condition that $s>2 \mu$ must hold. This, note, is the condition for the existence of mutation-selection equilibrium. We therefore conclude that if a mutation-selection equilibrium exists, the modifier will always spread if it is perfectly linked. Considering the other extreme of free recombination, where $r=0.5$, we obtain the condition

$H>\frac{1-3 \mu}{2-\mu(6+s)+s}$.

For small values of $\mu$, this approximates to $1 /(2+s)$. For the case where $s$ is small, this is equivalent to the condition, derived above $\left(\S \S 2\right.$ ), that $H>1 / 2$ (i.e. $\epsilon>-s^{2}$ ) must hold. This confirms the verbal argument that homogenization is beneficial in the absence of strong synergistic epistasis between the genes. As $s$ increases, the necessary minimum value of $H$ approaches $1 / 3$.

These results may be understood by considering that the modifier spreads due to linkage disequilibrium between itself $(M)$ and the advantageous $A A$ pair. Conversion initially forces disequilibrium. Selection against the deleterious allele strengthens the disequilbrium, while recombination weakens it. Hence, low rates of recombination and high values of $s$, both favour homogenization. The verbal argument presented in $\$ 2$ misses the alteration of linkage disequilbrium due to the action of selection and recombination. Hence, the simple fitness argument is only in agreement with the modifier analysis when $r=0.5$ and $s$ is small.

\section{(b) The costly modifier}

When invasion is possible, simulation reveals that it is fastest when linkage is tight. As might then be expected, if modification is costly $(\varphi>0)$, tight linkage will be favoured (figure 2). Indeed, if modification is costly, even if linkage is perfect, the condition for the invasion of the modifier resolves simply to

$\varphi<\frac{\mu}{1-\mu}$.

So the maximum cost is of the order of the mutation rate and is independent of both $H$ and $s$. For higher recombination rates, the maximum cost is lower still (figure 2). Tight linkage between the modifier and the array will hence be selectively favourable. Alternatively, we might assume that the maximum cost that the modifier can suffer is proportional to the strength of selection favouring an otherwise cost-free modifier. This being so, we might conclude that if selection on the modifier is weak (when $\mu$ is low and $s$ is low) and drift can thus oppose the invasion of the modifier, tight linkage between the modifier and the array will be necessary for the spread of the modifier.

\section{LINKAGE BETWEEN THE VIABILITY LOCI}

So far we have assumed that the two viability loci $\left(V_{1}\right.$ and $V_{2}$ ) are perfectly linked in an array. What if 


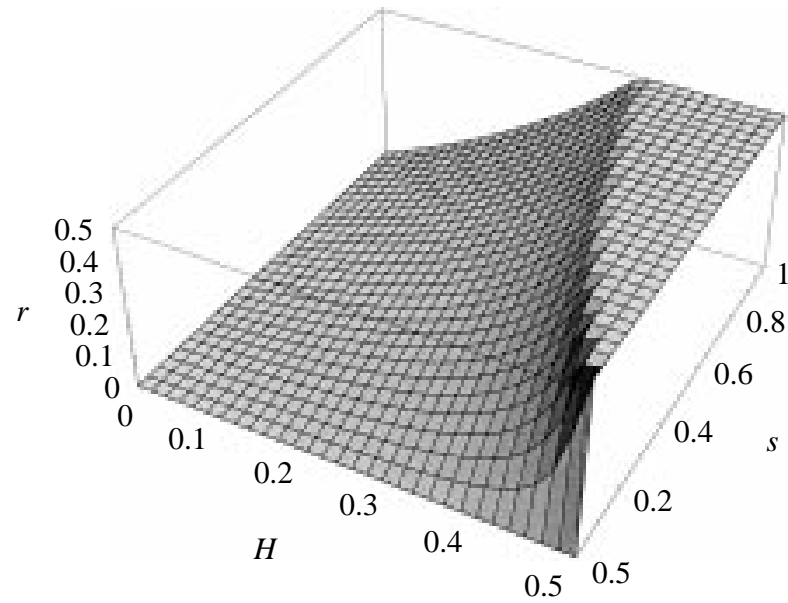

Figure 1. The maximum value of the recombination rate between the modifier and the array, consistent with the invasion of the neutral modifier causing conversion, as a function of effective penetrance $(H)$ of the mutant version of the allele and the selective coefficient $(s)$. This is for the example $s=0.1$ and for $\mu=0.0001$. Note that as $H$ decreases, so the necessity for tight linkage becomes all the more important. Likewise, as $s$ decreases, so does the necessity for linkage increase. These results were confirmed by simulation. The form of the curves is not very sensitive to variation in $\mu$, so long as a mutationselection equilibrium exists.

recombination could occur between these loci as well? Will this make the invasion conditions of the modifier more or less stringent? To investigate this problem we have extended the recursions to allow for recombination between the two viability loci (see Appendix 1).

Our simulations produce two notable conclusions (figure 3). If recombination between the modifier and the first viability locus $\left(V_{1}\right)$ is free $\left(r_{1}=0.5\right)$, then recombination $\left(V_{2}\right)$ between the two viability loci makes no difference to the results. The analytical solutions given above for $r=0.5$ are therefore applicable. This is intuitively reasonable. If there is free recombination between $M$ and $V_{1}$, then recombination between $V_{1}$ and $V_{2}$ cannot make the system even more randomized. However, if recombination between the modifier and the first viability locus is not free (i.e. $r_{1}<0.5$ ), then lower values of $r_{2}$ ease the invasion of the modifier. Invasion is easiest when $r_{1}=r_{2}=0$, i.e. the case of perfect linkage given above. We conclude that if homogenization is favoured, selection will also favour linkage between the genes.

\section{DISCUSSION}

\section{(a) Conclusions and predictions}

It has been shown that a cost-free modifier controlling the ability of an array to homogenize can spread under particular conditions. If one mutation has little effect, but the second mutation drastically reduces fitness, then a subtle conflict of interests occurs: closely linked modifiers favour homogenization while more distant modifiers do not. If, however, one mutation has a large effect, proportional to that of the second, then a modifier can invade regardless of linkage. Invasion is most likely if the mutations are of large effect. If the modifier is costly, then

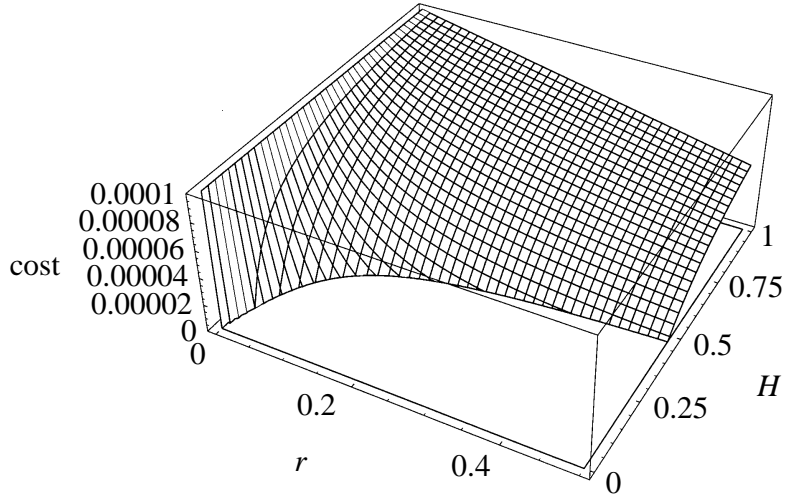

Figure 2. The maximum cost of modification consistent with invasion of the modifier, as a function of the recombination rate $(r)$, and penetrance $(H)$. For this example $\mu=0.0001$ and $s=0.1$.

tight linkage and antagonism between mutations both increase the probability of invasion.

These results can be contrasted with the analysis of the effects of homogenization on load (Ohta 1989) which find that homogenization always reduces the load. In the longterm, then, homogenization is uniformly beneficial to a lineage. Note that the analysis of the perfectly linked modifier is essentially the same as the load argument, and hence both agree that homogenization is beneficial. The modifier analysis shows, however, that homogenization is beneficial in the short-term only under special conditions, i.e. only if interactions between deleterious mutations are not strongly synergistic can the modifier take any linkage arrangement with the array.

We have not considered the effects of homogenization on advantageous alleles. The condition that deleterious mutations must not be strongly synergistic, could alternatively be stated, as regards advantageous mutations, that homogenization may be adaptive if most advantageous mutations are recessive (see, also, Slatkin 1986). We may therefore expect that homogenization will promote the spread of advantageous recessives (for a possible example, see Inomata \& Yamazaki 1996). Given, however, that the analysis for a recessive advantageous gene will depend greatly on ploidy, we shall not discuss the problem further.

From the above conclusions we can attempt to formulate predictions about which organisms, and which genes in those organisms, are most likely to undergo homogenization. Whether the load arguments make any qualitatively different predictions to the modifier argument depends critically on what the modifiers are and how they are linked to the genes being modified.

(i) Prediction 1. Homogenization should be most frequent in genes for which mutations are dominant

That a freely recombining modifier of homogenization is favoured by selection when interactions between mutations are not strongly synergistic may provide the strongest prediction of the model. This condition, that $H<1 / 2$, is equivalent to the case, in the normal diploid condition, for a mutation to be partially or wholly dominant. However, highly deleterious mutations tend to be recessive and slightly deleterious ones tend to be partially 


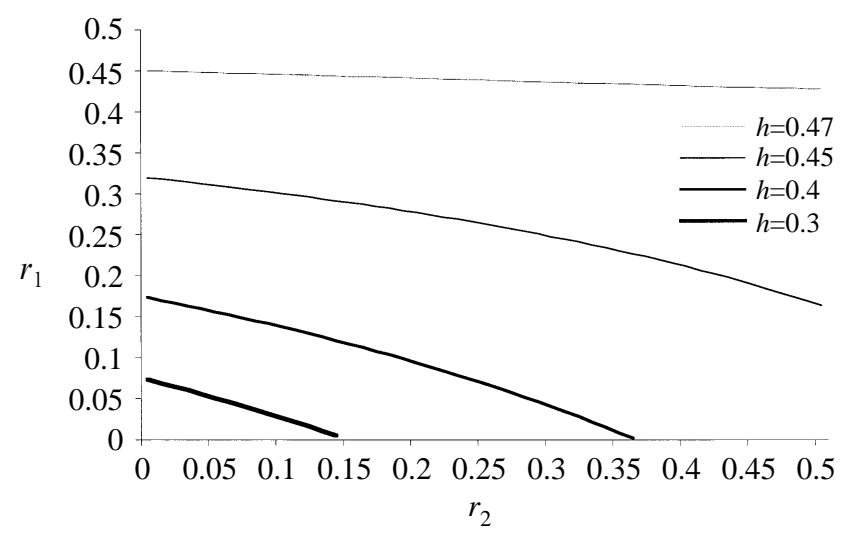

Figure 3. Critical minimum values for the rate of recombination between the modifier and the first viability locus $\left(r_{1}\right)$ and between the two viability loci $\left(r_{2}\right)$ for four values of the penetrance $(H)$. These plots are for the condition where $\mu=0.0001$ and $s=0.1$. These results were obtained by simulation. The simulations were allowed to run to a mutation-selection equilibrium prior to the introduction of a rare modifier. The modifier is introduced in linkage equilibrium.

recessive (Kacser \& Burns 1981). The necessary conditions seem not then to apply to most deleterious mutations. From this we conclude either (1) that homogenization is not adaptive in the short-term, or (2) that the modifiers are usually linked, or (3) that multicopy genes that become homogenized are an unusual class of genes in which mutations may often be at least partially dominant.

It is difficult at present to arbitrate between the three possibilities. However, we wish to suggest that the third possibility may be correct. While most mutations are recessive, mutagenesis studies in many organisms indicate that possibly as many as $10 \%$ are dominant or semidominant (Wilkie 1994). Haploinsufficiency is implicated in at least 35 human disease syndromes (Fisher \& Scambler 1994) and at least 2000 human genetic disorders are recognized for which the phenotypic penetrance is non-zero (see Online Mendelian Inheritance in Man: http:// www3.ncbi.nlm.nih.gov/omim). Although referred to as dominant disorders, $h$ is rarely, if ever, equal to unity. Often symptoms are apparent in heterozygotes, but not the full-blown symptoms as witnessed in the homozygotes. Note, however, that for the model the important value is the penetrance with respect to fitness. Heterozygotes may not have the full-blown disease, but in conditions of strong competition they may be liable to die or not reproduce. Their fitness may hence tend towards zero and $h$ (as regards fitness) will thus approach unity, while $h$, as regards phenotype, may be comparably low.

What sort of genes are likely to have high values of $h$ with respect to fitness, and are multicopy genes more prone to such mutations? Analysing values of $h$ pertinent to fitness rather than phenotype is difficult. We shall instead ask whether there are any generalizations about genes for which mutations are phenotypically dominant (or partially so)? We have searched the mouse knockout database (http://biomednet.com/ cgi-bin/mko/mkobrwse.pl) to find those genes for which the knockout is lethal in the heterozygous condition (i.e. the stringent case that $s=1$ and $h=1$ ). We found only eight (the database describes around 250 single-gene knockouts resulting in inviability and over 500 that are viable). These are: (1) core-binding factor alpha 2; (2) glutamate receptor, ionotropic, AMPA2 (alpha 2); (3) hepatocyte growth factor; (4) neural cell adhesion molecule, (5) orthodenticle homologue 2; (6) transforming growth factor, beta1; (7) vascular endothelial growth factor; and (8) mammalian achaete scute homologue 2 (Mash2). There are several more that have major phenotypic effects in heterozygotes, but which are not lethal.

All except one (core-binding factor) of the above eight genes affect growth in one form or another. This might not appear remarkable until one realizes that only about $4 \%$ of murine knockouts affect growth (Barlow 1995). There is therefore a vast excess of growth-associated genes in the dominant lethal knockout class. There may be a trivial explanation for this finding, namely the genes may be imprinted (hence half the time the knockouts will be hemizygotes for the knockout). One might suspect this given that there are more imprinted genes affecting growth than one would expect by chance (Barlow 1995). However, Mash2 is the only one in the list known to be imprinted (Guillemot $e t$ al. 1995). Knockouts of this are only inviable if maternally inherited. We know of no similar evidence to implicate any of the other genes in imprinting. Importantly, too, some genes known not to be imprinted do have major growth effects in the heterozygous knockout condition (e.g. IgfI; Liu et al. 1993). Of the 35 human disorders associated with haploinsufficiency (Fisher \& Scambler 1994), none are known to be associated with imprinting.

If the over-abundance of growth-associated genes in the set of dominant lethal knockouts is not due to their being imprinted, why else might this result be found? It seems reasonable to suppose that dosage sensitivity might be what one would expect a priori of genes with effects in the heterozygous condition (Wilkie 1994). There is considerable evidence that many growth factors are dosage dependent. Incremental over-expression of insulin-like growth factor II, shows a large and positively correlated response of embryonic size to dosage (Sun et al. 1997). Similarly, overexpression of growth factors (and their suppressors) frequently results in both alterations of dimensions and lethality (Hurst \& McVean 1997). We might then predict that dosage-sensitive genes, of which growth factors would be one important class, should more commonly undergo concerted evolution than others (for discussion of which genes might be dose sensitive, see Fisher \& Scambler 1994). One can make an anecdotal case that this might be true. Ribosomal DNA, splice factors, and ubiquitination genes all have been reported as undergoing homogenization (e.g. Hillis et al. 1991; Liao et al. 1997; Pavelitz et al. 1995; Schlotterer \& Tautz 1994; Zhou \& Ragan 1995) and are all produced at very high levels.

Assuming that dosage-sensitive genes are likely to benefit from homogenization, we can also predict that concerted evolution will probably be an extremely common phenomenon amongst repeat genes that share the same function. This comes from the understanding that selection will favour the duplication of a gene, if the effect of the gene is dosage dependent. Hence, we may expect, a priori, that multicopy genes are more likely to be those that are dose sensitive, and hence could benefit from homogenization. 
The above proposition is testable. Not all gene families need be dosage dependent. Some multicopy families do not have all members expressed in the same tissue. Instead, the numerous copies each have tissue-specific activity. We may then predict that gene families in which all (or most) copies are expressed synchronously in the same cell (e.g. rDNA) should have dominant mutations more often than those in which different members of the gene family have tissue-specific expression, and hence do not coexpress in the same cell.

\section{(ii) Prediction 2. Homogenization should be more common when recombination rates are low}

That invasion is always easiest when the array and the modifier are linked suggests that homogenization may be more common for arrays with low recombination rates and that selection may favour multigene families being in linked arrays. It also suggests that organisms with low recombination rates should have higher rates of homogenization. However, these may not be strong predictions, for two reasons. First, recombination may be necessary for homogenization to occur (unequal crossing over, for example, requires crossing over to occur). Second, advantageous mutations have a hard time spreading in asexual populations or in regions of low recombination (reviewed in Hurst \& Peck 1996). A modifier linked to an array in a region of low recombination might then be invisible to selection. These caveats aside, it is noteworthy that if one array has the ability to undergo homogenization, selection could favour the linkage of that array with others that would benefit from homogenization. Suggestively, this clumping of different arrays is a commonly evolved trait (Drouin \& de Sa 1995).

\section{(iii) Prediction 3. Homogenization should be most common in} organisms with large population sizes

The maximum cost that the modifier can suffer and still invade is of the order of the mutation rate of each gene in the array. This suggests that even if interactions are strongly antagonistic (dominant), if selection is weak, homogenization may not be favoured. As the efficiency of selection is dependent upon the population size, we may conclude that homogenization is more likely to be adaptive in species whose population size is large. Rough calculations (not shown) suggest that it is unlikely that mammalian populations are large enough to allow selection to favour a modifier affecting only one gene array. If, however, one modifier can affect many arrays simultaneously, then its spread may well be adaptive. Under this circumstance, however, it is unlikely that the modifier could be simultaneously linked to all arrays (but see Drouin \& de Sa 1995). Hence, there may be differences between taxa in the linkage of genes undergoing homogenization, with those in small populations being unlinked more commonly.

\section{(b) Homogenization, sex and ploidy}

As might perhaps be expected, our results are similar to those for the non-invasion of a modifier of diploidy entering a haploid population (Otto \& Goldstein 1992). Diploidy may be advantageous because deleterious mutations are masked. Conversely, haploidy and homogenization are favoured if masking is not adequately effective. Another way to read this conclusion is to suppose that a diploid organism can treat different genes differently. Those for which masking is beneficial can be left unhomogenized. Those for which masking is deleterious can be homogenized.

The theory outlined here is important in the context of theories of sex. Kondrashov (1988) has shown that if interactions between deleterious mutations are synergistic, then sex will be favoured, so long as the mutation rate is high enough, as it increases variance between progeny in the degree of genome contamination (see also Barton 1995; Charlesworth 1990; Kondrashov 1984). What, one might then ask, should an organism do if some interactions are synergistic but others are not (or only weakly so)? More generally, what will happen if there is variance in the form of the $\epsilon$, the parameter defining the form of the epistatic interaction? It has been shown that such variance reduces the parameter space in which recombination is beneficial (Otto \& Feldman 1997). For evidence of such variance see Elena \& Lenski (1997).

The analysis provided here goes some way to countering this objection to the mutational deterministic theory. Assuming that the genes interacting with $\epsilon>-s^{2}$ belong to the same gene family, by homogenizing these alone and preventing recombination between the viability loci, a sexual organism can minimize the deleterious effects of any mutations. In contrast, by promoting recombination and preventing homogenization, a sexual organism can minimize the effects of mutations that interact synergistically. It is as though part of the genome was sexual (not homogenized and recombining), and part was asexual (homogenized and not recombining). It seems then that with respect both to haploidy/diploidy and sexual/ asexual, the organism can, to coin a term, 'have its cake and eat it'.

We wish to thank Alexey Kondrashov, Eörs Szathmáry, and Gil McVean for comments on an earlier version of the manuscript. L.D.H. is funded by the Royal Society.

\section{APPENDIX 1}

Recursions for the three locus model. We consider a gene order $M V_{1} V_{2}$ where $V_{1}$ and $V_{2}$ are the two viability loci. Recombination occurs between $M$ and $V_{1}$ at a rate $r_{1}$, and between $V_{1}$ and $V_{2}$ at a rate $r_{2}$.

To avoid confusion with the original model we shall adopt a new nomenclature incorporating the haploid frequencies as terms in $z$ rather than $x$. There are eight haploid types prior to zygote formation, these being: $M A A$ at frequency $z_{1}, M A a$ at frequency $z_{21}, M a A$ at frequency $z_{22}, M a a$ at frequency $z_{3}, m A A$ at frequency $z_{4}$, $m A a$ at frequency $z_{51}, m a A$ at frequency $z_{52}$, and maa at frequency $z_{6}$. As in the above model, prior to zygote formation there are no heterozygotes associated with the modifier $\left(z_{21}=z_{22}=0\right)$. After sex and recombination the new genotype frequencies become:

$$
\begin{aligned}
z_{1 a}= & z_{1}\left[z_{1}+z_{1} z_{3}\left(1-r_{2}\right)+z_{4}+z_{51}\left(\left(1-r_{1}\right)\left(1-r_{2}\right)\right.\right. \\
& \left.\left.+r_{1} r_{2}\right)+z_{52}\left(1-r_{1}\right)+z_{6}\left(1-r_{1}\right)\left(1-r_{2}\right)\right] \\
& +z_{3} z_{4} r_{1}\left(1-r_{2}\right), \\
z_{21 a}= & z_{1}\left[z_{3} r_{2}+z_{51}\left(r_{1}\left(1-r_{2}\right)+\left(1-r_{1}\right) r_{2}\right)\right. \\
& \left.+z_{6}\left(1-r_{1}\right) r_{2}\right]+z_{3}\left[z_{4} r_{1} r_{2}+z_{51} r_{1}\right],
\end{aligned}
$$




$$
\begin{aligned}
z_{22 a}= & z_{1}\left[z_{3} r_{2}+z_{52} r_{1}+z_{6} r_{1} r_{2}\right]+z_{3}\left[z_{4}\left(1-r_{1}\right) r_{2}\right. \\
& \left.+z_{52}\left(r_{1}\left(1-r_{2}\right)+\left(1-r_{1}\right) r_{2}\right)\right], \\
z_{3 a}= & z_{1}\left[z_{3}\left(1-r_{2}\right)+z_{6} r_{1}\left(1-r_{2}\right)\right] \\
& +z_{3}\left[z_{3}+z_{4}\left(1-r_{1}\right)\left(1-r_{2}\right)+z_{51}\left(1-r_{1}\right)\right. \\
& \left.+z_{52}\left(\left(1-r_{1}\right)\left(1-r_{2}\right)+r_{1} r_{2}\right)+z_{6}\right], \\
z_{4 a}= & z_{1}\left[z_{4}+z_{51}\left(r_{1}\left(1-r_{2}\right)+\left(1-r_{1}\right) r_{2}\right)+z_{52} r_{1}\right. \\
& \left.+z_{6} r_{1}\left(1-r_{2}\right)\right]+z_{4}\left[z_{3}\left(1-r_{1}\right)\left(1-r_{2}\right)\right. \\
& \left.+z_{4}+z_{51}+z_{52}+z_{6}\left(1-r_{2}\right)\right]+z_{51} z_{52} r_{2}, \\
z_{51 a}= & z_{1}\left[z_{51}\left(\left(1-r_{1}\right)\left(1-r_{2}\right)+r_{1} r_{2}\right)+z_{6} r_{1} r_{2}\right] \\
& +z_{3} z_{4}\left(1-r_{1}\right) r_{2}+z_{51}\left[z_{3}\left(1-r_{1}\right)\right. \\
& \left.+z_{4}+z_{6}+z_{51}+z_{52}\left(1-r_{2}\right)+z_{6}\right], \\
z_{52 a}= & z_{1}\left[z_{52}\left(1-r_{1}\right)+z_{6}\left(1-r_{1}\right) r_{2}\right]+z_{4}\left[z_{3} r_{1} r_{2}+z_{6} r_{2}\right] \\
& +z_{52}\left[z_{3}\left(\left(1-r_{1}\right)\left(1-r_{2}\right)+r_{1} r_{2}\right)+z_{4}\right. \\
& \left.+z_{51}\left(1-r_{2}\right)+z_{52}+z_{6}\right], \\
z_{6 a}= & z_{3}\left[z_{4} r_{1}\left(1-r_{2}\right)+z_{51} r_{1}+z_{52}\left(r_{1}\left(1-r_{2}\right)\right.\right. \\
& \left.\left.+\left(1-r_{1}\right) r_{2}\right)+z_{6}\right]+z_{51} z_{52} r_{2}+z_{6}\left[z_{1}\left(1-r_{1}\right)\right. \\
& \left.\left(1-r_{2}\right)+z_{3}+z_{4}\left(1-r_{2}\right)+z_{51}+z_{52}+z_{6}\right] .
\end{aligned}
$$

After this the recursions are trivial adaptations of those given in the text and so not presented. A $a$ types have the same fitness and mutation rates as $a A$ types, i.e. gene order does not affect these parameters. For results of the simulations see figure 3.

\section{REFERENCES}

Barlow, D. P. 1995 Gametic imprinting in mammals. Science 270, 1610-1613.

Barton, N. H. 1995 A general-model for the evolution of recombination. Genet. Res. 65, 123-144.

Bengtsson, B. O. 1990 The effect of biased conversion on the mutation load. Genet. Res. 55, 183-187.

Bodmer, W. F. \& Parsons, P. A. 1962 Linkage and recombination in evolution. Adv. Genet. 11, 1-100.

Charlesworth, B. 1990 Mutation-selection balance and the evolutionary advantage of sex and recombination. Genet. Res. 55, 199-221

Charlesworth, B. \& Hartl, D. L. 1978 Population dynamics of the segregation distorter polymorphism of Drosophila melanogaster. Genetics 89, 171-192.

Drouin, G. \& de Sa, M. M. 1995 The concerted evolution of 5S ribosomal genes linked to the repeat units of other multigene families. Molec. Biol. Evol. 12, 481-493.

Elder, J. F. \& Turner, B. J. 1995 Concerted evolution of repetitive DNA sequences in eukaryotes. O. Rev. Biol. 70, 297-320.

Elena, S. F. \& Lenski, R. E. 1997 Test of synergistic interactions among deleterious mutations in bacteria. Nature 390, 395-398.

Fisher, E. \& Scambler, P. 1994 Human haploinsufficiency-one for sorrow, two for joy. Nat. Genet. 7, 5-7.

Guillemot, F., Caspary,T.,Tilghman, S. M., Copeland, N. G., Gilbert, D. J., Jenkins, N. A., Anderson, D. J., Joyner, A. L., Rossant, J. \& Nagy, A. 1995 Genomicimprinting of Mash2, a mouse gene required for trophoblast development. Nat. Genet. 9, 235-242.

Haig, D. 1993 Genetic conflicts in human pregnancy. Q. Rev. Biol. 68, 495-532.
Hillis, D. M., Moritz, C., Porter, C. A. \& Baker, R. J. 1991 Evidence for biased gene conversion in concerted evolution of ribosomal DNA. Science 251, 308-310.

Hurst, L. D. 1992 Is Stellate a relict meiotic driver? Genetics 130, 229-230.

Hurst, L. D. 1994 Embryonic growth and the evolution of the mammalian $\mathrm{Y}$ chromosome. I. The $\mathrm{Y}$ as an attractor for selfish growth factors. Heredity 73, 223-232.

Hurst, L. D. 1996 Further evidence consistent with Stellate's involvement in meiotic drive. Genetics 142, 641-643.

Hurst, L. D. \& McVean, G. T. 1997 Growth effects of uniparental disomies and the conflict theory of genomic imprinting. Trends Genet. 13, 436-443.

Hurst, L. D. \& Peck, J. R. 1996 Recent advances in understanding the evolution and maintenance of sex. Trends Ecol. Evol. 11, 46-52.

Hutson, V. \& Law, R. 1993 Four steps to two sexes. Proc. R. Soc. Lond. B 253, 43-51.

Inomata, N. \& Yamazaki, T. 1996 Adaptive evolution at the molecular level of the duplicated AMY gene system in Drosophila. 7. Genet. 75, 125-137.

Kacser, H. \& Burns, J. A. 1981 The molecular basis of dominance. Genetics 97, 639-666.

Kondrashov, A. 1988 Deleterious mutations and the evolution of sexual reproduction. Nature 336, 435-440.

Kondrashov, A. S. 1984 Deleterious mutations as an evolutionary factor. I. The advantage of recombination. Genet. Res. 44, 199-217.

Korol, A. B., Preigel, I. A. \& Preigel, S. I. 1994 Recombination variability and evolution. London: Chapman \& Hall.

Liao, D. Q., Pavelitz, T., Kidd, J. R., Kidd, K. K. \& Weiner, A. M. 1997 Concerted evolution of the tandemly repeated genes encoding human U2 snRNA (the RNU2 locus) involves rapid intrachromosomal homogenization and rare interchromosomal gene conversion. EMBO f. 16, 588-598.

Liu, J. P., Baker, J., Perkins, A. S., Robertson, E. J. \& Efstratiadis, A. 1993 Mice carrying null mutations of the genes encoding insulin-like growth factor-I $(I g f-I)$ and type-1 Igf receptor $($ Igflr). Cell 75, 59-72.

Lyttle, T. W. 1991 Segregation distorters. A. Rev. Genet. 25, 511-557.

Ohta, T. 1989 The mutational load of a multigene family with uniform members. Genet. Res. 53, 141-145.

Otto, S. P. \& Feldman, M. W. 1997 Deleterious mutations, variable epistatic interactions, and the evolution of recombination. Theor. Popul. Biol. 51, 134-147.

Otto, S. P. \& Goldstein, D. B. 1992 Recombination and the evolution of diploidy. Genetics 131, 745-751.

Pavelitz, T., Rusche, L., Matera, A. G., Scharf, J. M. \& Weiner, A. M. 1995 Concerted evolution of the tandem array encoding primate U2 snRNA occurs in situ, without changing the cytological context of the RNU2 locus. EMBO 7. 14, 169-177.

Rice, W. R. 1992 Sexually antagonistic genes-experimental evidence. Science 256, 1436-1439.

Schlotterer, C. \& Tautz, D. 1994 Chromosomal homogeneity of Drosophila ribosomal DNA arrays suggests intrachromosomal exchanges drive concerted evolution. Curr. Biol. 4, 777-783.

Slatkin, M. 1986 Interchromosomal biased gene conversion, mutation and selection in a multigene family. Genetics 112, 681-698.

Sun, F. L., Dean, W., Kelsey, G., Allen, N. \& Reik, W. 1997 Transactivation of IGF2 results in Beckwith Wiedemann syndrome phenotypes. Nature 389, 809-815.

Weir, B. S., Ohta, T. \& Tachida, H. 1985 Gene conversion models. F. Theor. Biol. 116, 1-8.

Wilkie, A. O. M. 1994 The molecular basis of genetic dominance. 7. Med. Genet. 31, 89-98.

Zhou, Y. H. \& Ragan, M. A. 1995 Characterization of the polyubiquitin gene in the marine red alga Gracilaria verrucosa. Biochem. Biophys. Acta 1261, 215-222. 
